\title{
ENVIRONMENTAL EXTREMISTS IN THE VIRGO CLUSTER
}

\author{
JEFFREY KENNEY AND REBECCA KOOPMANN \\ Yale University, Astronomy Department, \\ P.O. Box 208101, New Haven, CT 06520-8101 USA
}

\section{Introduction}

Many types of galaxy interactions have been posited to occur in clusters, although it remains unclear which processes actually occur, and which ones might help explain the tendency for early type galaxies to inhabit high density environments, or cause the rapid evolution of cluster galaxies (e.g., Dressler et al. 1997). With these questions in mind, we have been conducting an environmental inventory of galaxies in the Virgo Cluster. Our approach is to combine surveys of spirals and S0s with detailed studies of the most interesting and peculiar galaxies. In this paper, we describe two main points. 1.) There is a population of spiral galaxies in the Virgo cluster with the small central light concentrations (bulge-to-disk ratios, or $\mathrm{B} / \mathrm{D}$ 's) characteristic of isolated $\mathrm{Sb}$ and $\mathrm{Sc}$ galaxies, but global star formation rates lower than those of isolated spirals of any Hubble class ( $\mathrm{Sa}-\mathrm{Sc}$ ). These Virgo galaxies are generally classified as "early type" (e.g. Sa), and thus contribute to the morphology-density relationship. 2.) There are several types of environmental interactions occurring in Virgo, including low velocity tidal interactions and mergers, high velocity tidal interactions and collisions, HI accretion, and ICM-ISM stripping. We discuss examples of some of these interactions.

\section{The Comparative Study of Cluster and Isolated Galaxies}

It is important to compare Virgo and isolated galaxies in a manner independent of subjective galaxy classification. From a data set of $\mathrm{R}$ and $\mathrm{H} \alpha$ images of nearly 100 Virgo cluster and isolated spiral galaxies, we have measured objective parameters to test how well the Hubble system succeeds in distinguishing between galaxies with different physical characteristics in 


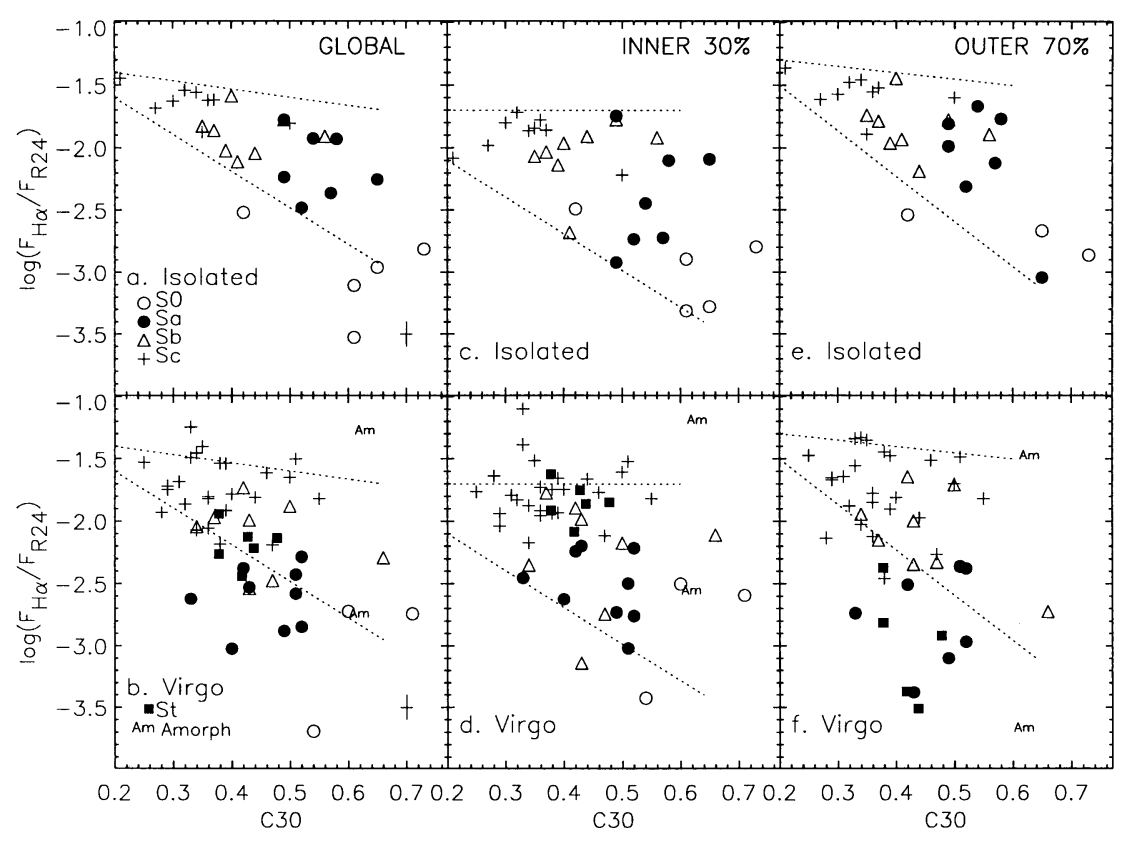

Figure 1. H $\alpha$ to $\mathrm{R}$ flux ratio vs. central R light concentration for isolated (top) and Virgo (bottom) galaxies. Vertical axis shows flux ratio for (left) entire galaxy, (middle) central $30 \%$ of optical disk, and (right) outer $70 \%$ of optical disk. There are good correlations for isolated Sa-Sc's, but poor correlations for Virgo Sa-Sc's. Lines bound the regions in which isolated Sa-Sc's lie. Peculiar Virgo spirals are labeled St. Most isolated Sa's tend to be high concentration systems, while most Virgo Sa's are low concentration systems with low star formation rates. Star formation is normal or enhanced in the centers of Virgo galaxies, and strongly depleted in the outer disks (Koopmann \& Kenney 1998a,b).

the two environments (Koopmann \& Kenney 1998a,b). These results are important for understanding the morphology-density relationship, and also demonstrate that Hubble classification is meaningful for isolated spirals, but not for most cluster spirals.

We measure parameters which trace two of the criteria used for Hubble classification: the central light concentration in R (C30), a tracer of the $\mathrm{B} / \mathrm{D}$, and the $\mathrm{H} \alpha$ to $\mathrm{R}$ flux ratio $\left(\frac{F_{\mathrm{H} \alpha}}{F_{\mathrm{R} 24}}\right)$, a tracer of the star formation rate and the "knottiness" of spiral arms. These parameters are well correlated for isolated galaxies, as shown in Fig. 1a. Assigned Hubble types, indicated by different symbols, correlate well with both parameters. Note especially that $\mathrm{Sa}$ and Sc galaxies are distinguished by both their central light concentrations and their star formation rates in a manner consistent with their Hubble classifications. The situation is very different for the Virgo galaxies (Fig. 1b). C30 and $\frac{F_{\mathrm{H} \alpha}}{F_{\mathrm{R} 24}}$ are virtually uncorrelated, and there is a greater 

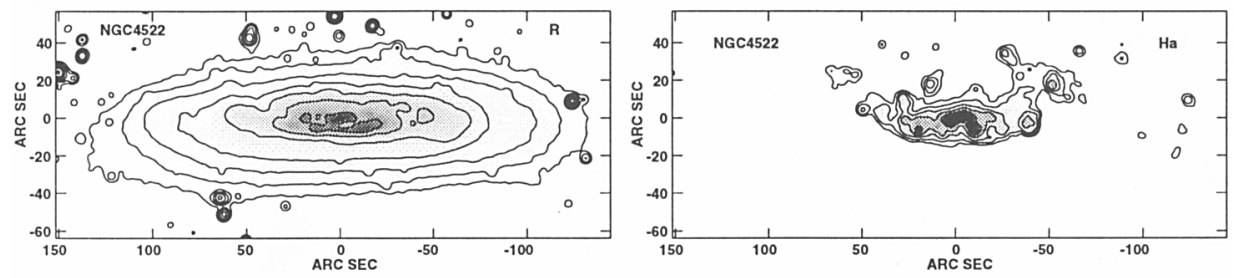

Figure 2. (left) R image of NGC 4522 shows fairly regular isophotes at large radii, indicating that the stellar disk is undisturbed. (right) $\mathrm{H} \alpha$ image shows truncated star-forming disk, and extraplanar HII regions. This combination suggests that NGC 4522 is presently experiencing a strong ICM-ISM interaction. From Kenney \& Koopman (1998).

range of star formation rates at every central light concentration, compared to the isolated sample. While the Sa and Sc galaxies are still well separated, they are distinguished principally by their star formation rates rather than their central light concentrations. Many of the Virgo Sa galaxies are actually systems with the low central light concentrations characteristic of isolated Sb-Sc galaxies. Fig. 1c-f show that it is primarily the outer disks of Virgo galaxies which have low star formation rates. Most Virgo galaxies are truncated rather than globally anemic. This strongly suggests that environmental processes remove gas from the outer disks of cluster spirals of all B/Ds, and those that lose gas become classified as early type spirals due to the resulting decrease in their star formation rate. This has implications for the morphology-density relationship, as well as other comparisons of cluster and field spirals. Part of the excess of early type galaxies in nearby clusters is due to small $B / D$ systems with reduced SFRs. It is not all due to a systematic change in $B / D$ with environmental density.

\section{Examples of Different Environmental Processes}

\subsection{NGC 4522 AND ICM-ISM STRIPPING}

The stripping of gas from the interstellar medium (ISM) of galaxies due to interactions with the gas in the intracluster medium (ICM) may be one of the most important types of interactions which occur in clusters of galaxies. Although many galaxies have properties consistent with having been stripped, there have been no clear examples of gas actively being stripped from the disks of spirals. The highly inclined Virgo cluster spiral galaxy NGC 4522 may be such a case. Our R-band and $\mathrm{H} \alpha$ images from the WIYN telescope, shown in Figure 2, reveal a relatively undisturbed stellar disk and a bow-shock-shaped $\mathrm{H} \alpha$ morphology, which strongly suggest that the ISM of NGC 4522 is being stripped by the gas pressure of the ICM. The presence of HII regions apparently located above the disk plane suggests 

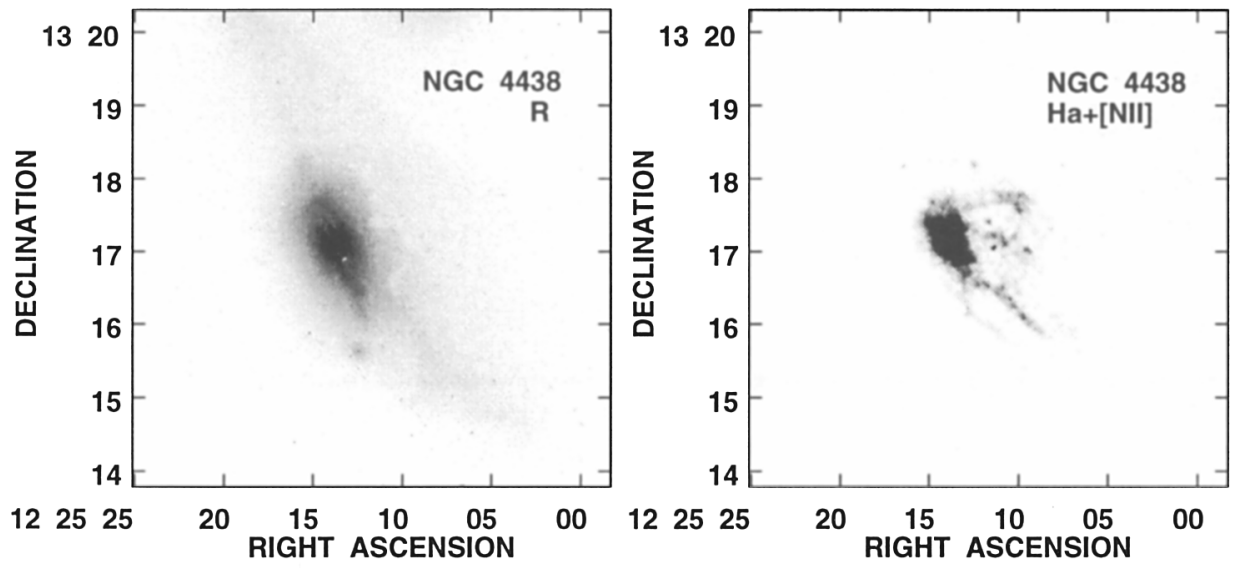

Figure 3. R (left) and $\mathrm{H} \alpha+[\mathrm{NII}]$ (right) maps of NGC 4438, showing disturbed stellar disk and remarkable ionized gas filaments. The morphology and kinematics of this gas suggest that the gas is cooling and falling into the main body of NGC 4438 . We propose that this type of disturbed ISM results from the aftermath of a high-velocity ISM-ISM collision. From Kenney et al. (1995).

that star formation is occurring in the stripped gas, and that newly formed stars will enter the galaxy halo and/or intracluster space.

\subsection{NGC 4438 AND HIGH-VELOCITY COLLISIONS}

Close interactions which occur at velocities too high to result in merging also disturb a galaxy's structure. Broadband images of NGC 4438 (Fig 3) show a highly disturbed edge-on stellar disk, with stellar debris displaced to the west of the galaxy's main disk. Simulations are able to produce this type of disturbed stellar morphology with a high velocity $\left(\sim 1000 \mathrm{~km} \mathrm{~s}^{-1}\right)$ interaction between galaxies of similar mass. Combes et al. (1988) suggest a collision with a closest approach of $\sim 5 \mathrm{kpc}$ with the nearby galaxy NGC 4435 , although Moore et al. (1996) suggest a more distant encounter at $\sim 30 \mathrm{kpc}$ with an unidentified galaxy. Our multiwavelength study of NGC 4438 shows that the ISM in this galaxy is also severely disturbed (Kenney et al. 1995). The $\mathrm{H} \alpha+[\mathrm{NII}]$ image (Fig. 3) reveals several remarkable ionized gas filaments which extend $\sim 5-10 \mathrm{kpc}$ from a gas-rich nuclear disk to a second gas-rich region with strong [NII], x-ray, radio continuum, $\mathrm{CO}$ and $\mathrm{HI}$ emission. Spectroscopy shows that these filaments have low velocities and line ratios similar to those in "cooling flow" galaxies, which suggests that the filament gas is falling back into the disk and cooling. The ISM in NGC 4438 is much more heavily disturbed than in NGC 4522 , and we propose that this results from a high velocity ISM-ISM collision between NGC 4438 and 

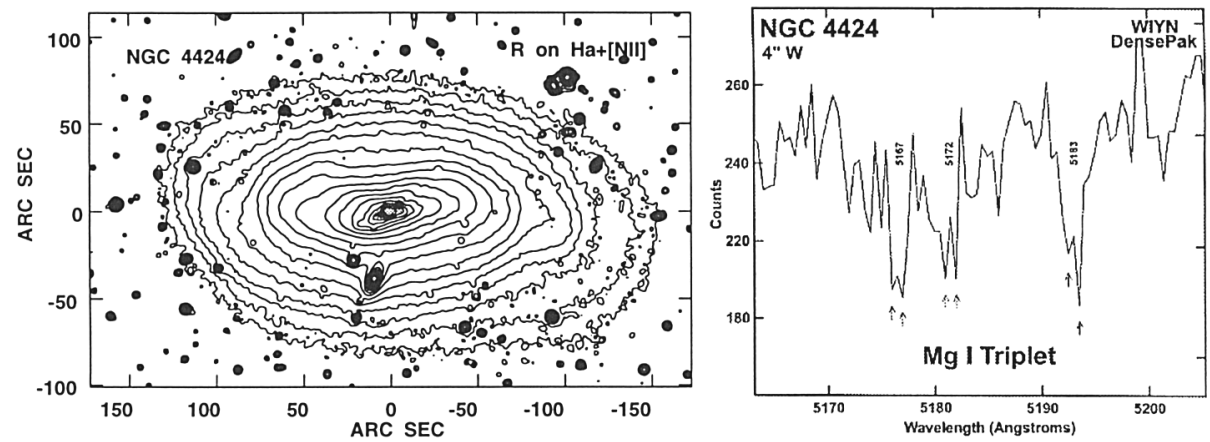

Figure 4. (left) $\mathrm{R}$ contour and $\mathrm{H} \alpha+[\mathrm{N}$ II] grayscale plot of NGC 4424, a likely intermediate-mass-ratio merger remnant. Note heart-shaped contours in $\mathrm{R}$ and confinement of $\mathrm{H} \alpha$ emission to the central $10^{\prime \prime}$. From Kenney et al. (1996). (right) Portion of optical spectrum $4^{\prime \prime} \mathrm{W}$ of nucleus in NGC 4424, obtained with the WIYN Densepak fiber array spectrograph. Note that each component of the $\mathrm{Mg}$ triplet is doubled, indicating cospatial, counterrotating stellar components. From Winnick \& Kenney (in prep).

another galaxy. Therefore closest approach was probably less than $30 \mathrm{kpc}$, although the lack of any obvious ring-like structure in NGC 4438 suggests that closet approach may have been greater than $5 \mathrm{kpc}$.

\subsection{NGC 4424 AND MERGERS}

Mergers do not occur at the high velocities which characterize many of the interactions that occur in clusters. Yet there may be enough low velocity interactions in clusters to make merging an important process for transforming spirals into S0s. Our studies of the peculiar (Sa pec) Virgo cluster galaxy NGC 4424 indicate that this system is the product of a merger (Kenney et al. 1996). The $\mathrm{R}$ image reveals banana-shaped isophotes, shelllike features and other complex structure generally associated with mergers (Fig. 4a). The only $\mathrm{H} \alpha$ emission arises from a few bright HII complexes distributed along a bar-like feature located within $500 \mathrm{pc}$ of the nucleus, inside the bulge-dominated region. Spectra reveal double-peaked stellar absorption lines (Fig. 4b), indicating the presence of cospatial, counterrotating stars in the circumnuclear region. These peculiar properties strongly suggest a recent, intermediate mass ratio (0.1-0.5) merger, and we propose that the galaxy will become a small-bulge S0 within $\sim 1$ Gyr.

\section{References}

Combes, F., Dupraz, C., Casoli, F., \& Pagani, L. (1988), AA, 203, L9.

Dressler, A., Oemler, A., Couch, W. J., Smail, I., Ellis, R. S., Barger, A., Butcher, H., Poggianti, B. M., \& Sharples, R. M. 1997, ApJ, in press. 
Kenney, J. D. P, Rubin, V. C., Planesas, P., \& Young, J. S. (1995), ApJ, 438, 135.

Kenney, J.D.P. \& Koopmann, R.A. (1998), ApJL, in prep.

Kenney, J.D.P., Koopmann, R. A., Rubin, V., \& Young, J. S. (1996), AJ, 111, 152.

Koopmann, R.A. \& Kenney, J.D.P. (1998a), ApJL, 000, submitted.

Koopmann, R.A. \& Kenney, J.D.P. (1998b), ApJ, 000, in prep.

Moore, B., Katz, N., Lake, G., Dressler, A., \& Oemler, A., Jr. (1996), Nature, 379, 613. 